\title{
Does presenting with meconium ileus affect the prognosis of children with cystic fibrosis?
}

\begin{tabular}{|r|l|}
\hline Journal: & Pediatric Pulmonology \\
\hline Manuscript ID: & PPUL-09-0148.R2 \\
\hline Wiley - Manuscript type: & Original Article \\
\hline Date Submitted by the \\
Author: & 18-Apr-2010 \\
\hline Complete List of Authors: & $\begin{array}{l}\text { Johnson, Jo-Anne; University of Cambridge, Gurdon Institute } \\
\text { Bush, Andrew; Royal Brompton Hospital } \\
\text { Buchdahl, Roger; Hillingdon Hospital NHS Trust, Department of } \\
\text { paediatrics }\end{array}$ \\
\hline Keywords: & Lung function, Growth, Presentation, New born screening, Survival \\
\hline
\end{tabular}

\section{今 scholarONE" \\ Manuscript Central}




\section{Title page}

Does presenting with meconium ileus affect the prognosis of children with cystic fibrosis?

${ }^{1}$ Jo-Anne Johnson MBChB (drjojohnson@ doctors.org.uk)

${ }^{2}$ Andrew Bush MD (A.Bush@rbh.nthames.nhs.uk)

${ }^{2}$ Roger Buchdahl MD (rbuc@easynet.co.uk)

${ }^{1}$ Wellcome Trust/Cancer Research UK Gurdon Institute, University of Cambridge, Tennis Court Road, Cambridge, CB2 1QN

${ }^{2}$ Royal Brompton and Harefield NHS Trust, Sydney Street, London, SW6 6NP

Correspondence to:

Professor Andrew Bush

Consultant Respiratory Paediatrician,

Royal Brompton Hospital

Sydney Street

London

SW6 3NP

United Kingdom

Fax 01895279581

E-mail a.bush@rbh.nthames.nhs.uk

Research conducted at Royal Brompton Hospital, Sydney Street, London, SW6 6NP

\section{Short Title}

Meconium ileus and outcome in cystic fibrosis 


\begin{abstract}
Summary
It is a matter of debate as to what extent the long-term outcome of cystic fibrosis (CF) is affected by presenting with meconium ileus (MI). We compared long-term clinical outcomes of CF children who presented with MI, to those presenting with other symptoms (non-MI) in an era of non new-bornscreening (NBS).
\end{abstract}

We collected annual lung function data between the ages of 8 to 15 years in terms of percent predicted first second forced expired volume (FEV1\%pr), percent predicted forced vital capacity (FVC\%pr), and between the ages of 2 to 15 years annual height and weight $\mathrm{z}$-scores ( $\mathrm{HtZ}$ and $\mathrm{WtZ}$ respectively) for children attending the Royal Brompton Hospital CF clinic. To be included in the study, subjects had to have at least 5 pulmonary function tests and 5 anthropometric measurements recorded over this period.

$38 \mathrm{MI}$ and 76 non-MI subjects were compared. There were no significant differences in genotype, sex, chronic Pseudomonas infection, or pancreatic enzyme use between the 2 groups. The median age of diagnosis was 1 day (MI) vs. 7 months (non-MI). There was a decline in spirometry and anthropometric variables over the study period for both MI and non-MI groups apart from WtZ score in the non-MI group. Mixed model analysis adjusting for potential confounders including genotype, pancreatic status, sex, chronic Pseudomonas aeruginosa lung infection, and age of diagnosis revealed no difference between the 2 groups in terms of lung function and growth during the time period of the study, however there was a non-significant trend for subjects presenting with MI to do better in all 4 parameters.

We conclude that babies presenting with MI have no worse long-term outcome than those presenting symptomatically later in infancy, despite having undergone invasive procedures in the newborn period. This underscores the importance of early diagnosis and treatment in $\mathrm{CF}$. 


\author{
Keywords \\ Lung function \\ Growth \\ Presentation \\ New born screening \\ Survival
}




\section{Introduction}

Meconium ileus (MI) is characterised by neonatal intestinal obstruction caused by the accumulation of desiccated and inspissated intraluminal meconium[1]. Around $15 \%$ of patients with pancreatic insufficient cystic fibrosis (CF) present with MI [2] but MI is also recorded rarely in normals and pancreatic sufficient CF [3]. It is a matter of debate as to what extent long-term outcome in CF is affected by MI.

Two groups from areas where new born screening (NBS) is established have suggested that patients presenting with MI have a worse prognosis in terms of lung function than those without MI diagnosed through NBS. [4,5,6,7] One of these groups, the Wisconsin group [5,6,7] also found MI patients had poorer growth.

However, 3 studies have suggested that MI patients have the same prognosis as patients presenting with other symptoms, not diagnosed through new-born-screening. Munck et al [8] in 2006 compared 26 children presenting with MI diagnosed with CF at a median age of 1 month to 26 children without MI presenting with other symptoms (unscreened) diagnosed with $\mathrm{CF}$ at a median age of 3.5 months. They compared height and weight $\mathrm{z}$ scores at 1,3,6,10 and 15 years of age. They also compared forced vital capacity (FVC) and first second forced expired volume (FEV1) at 6, 10 and 15 years. There was no difference between the two groups in growth or lung function and they concluded that presenting with MI made no difference to clinical outcome compared to presenting with other symptoms.

The second study [9] compared 158 patients presenting with MI to $1175 \mathrm{CF}$ patients presenting with other symptoms again in a pre-new born screening era. They compared 2 subgroups based on date of birth and showed that MI patients born between 1963 and 1972 had lower weight and height percentiles at age 13 years compared to non-MI patients. However, subjects born between 1973 and 1982 showed no difference in height and weight at 13 years. There were also no differences in FVC, FEV1, or forced expiratory flow at $25 \%$ to $75 \%$ of $\mathrm{FVC}\left(\mathrm{FEF}_{25-75)}\right.$ in this subgroup. There was a significantly better survival in the later compared to the earlier cohort, particularly in the MI group in 
the first year of life. This study suggests that advances in medical care especially in the perinatal period improved the prognosis of patients in the MI group.

The merits of the study by Kerem et al [9] include the large sample size and long time period of the study. However, the problem with such a long time period is the changes in medical practice taking place in this time. Although the authors tried to limit these differences by analysing the results in 2 cohorts, this resulted in a lack of pulmonary function test data to power the study.

The most recent study [10] retrospectively compared $60 \mathrm{CF}$ subjects with a history of MI (mean age of diagnosis 0.4 months) to 310 without MI presenting with other symptoms (mean age of diagnosis 21.8 months). Using a combination of cross-sectional and longitudinal analyses, they showed that there was no difference in lung function between the 2 groups. Although this study is more recent, the large age range of the study subjects ( $0-48$ years) means that it is highly likely that there was wide variation over time in the medical care that the patients received.

The aim of our single centre study is to test the hypothesis that with current standards of medical care and in an era of non-NBS there is no difference in clinical outcome between CF patients presenting with MI and patients without MI presenting with other symptoms.

The question has important practical as well as theoretical implications. If patients presenting with MI have the same or better prognosis than those diagnosed later with other symptoms, despite in many cases having had to undergo a major abdominal operation, this would be a pointer to the benefits of early diagnosis of $\mathrm{CF}$, which has been disputed by some [11]. On the other hand, if they do worse, despite modern treatment, this implies that new treatment strategies are needed in this group and that they would have potentially more to gain from novel, disease-modifying therapies. 


\section{Material and Methods}

Study Subjects:

CF patient data is updated on the Royal Brompton Hospital CF database after the patients' annual review by the CF nurse or secretary. By the $1^{\text {st }}$ January 2008,665 subjects had been registered onto the database since it began in 1986. All had a diagnosis of CF confirmed with a positive sweat test (a chloride value $>60 \mathrm{mmol} / \mathrm{l}$ in two sweat samples) and/or had the presence of two recognised CF disease-causing genetic mutations. Of the 665 subjects 75 of these had presented with meconium ileus and 590 with non meconium ileus (non-MI) symptoms including respiratory symptoms and/or failure to thrive. To be included, subjects had to be at least 15 years old at the time of the study (i.e. born before $1^{\text {st }}$ January 1993). Subjects had to have a minimum of 5 recorded annual pulmonary function tests in terms of percent predicted first second forced expired volume (FEV1\%pr) and percent predicted forced vital capacity (FVC\%pr) from the age of 8 years. They were also required to have at least 5 recordings of annual height and weight $\mathrm{z}$-scores ( $\mathrm{HtZ}$ and $\mathrm{WtZ}$ respectively) from the age of 2 years. Pulmonary and anthropometric data was analysed up to and including the $15^{\text {th }}$ year of life. Ethical approval was obtained from the Royal Brompton Hospital Research Ethics Committee. From 16 years of age many of the subjects were referred to other adult CF tertiary centres so survival data was the only outcome measure recorded thereafter. These were obtained by telephone contact to the adult tertiary centres to which the children had been referred.

Study Design:

The primary objective of our study was to test the hypothesis that, in an era of non-NBS there is no difference in clinical outcome between CF patients presenting with MI (MI group) and those without MI presenting with other symptoms (non-MI group). We used a retrospective longitudinal study to compare growth and pulmonary function data of eligible subjects in MI and non-MI groups on a yearby-year basis. Details of treatment protocols can be found at http://www.rbht.nhs.uk/healthprofessionals/clinical-depts/paediatrics/childrencf/

Methods/Statistical Analysis:

The primary outcomes were annual HtZ /WtZ scores (age 2-15 years) and FVC\%pr/ FEV1\%pr (age 815 years). 8 years was taken as the age at which pulmonary function testing could be accurately 
reproduced. Pulmonary function was measured using a Jaeger CompactLab pneumotachograph in the lung function laboratory by trained technicians. FEV1and FVC were measured and expressed as percentages of predicted values obtained from equations based on gender and height by Rosenthal et al [12]. Weight (minimal underclothing) and height (Harpenden stadiometer) were measured and expressed as standard deviation scores (Z-scores) using published population means and standard deviations [13]. Baseline characteristics of the study groups were compared using Chi Squared analysis with Fisher's exact test for categorical data and Mann-Whitney for continuous data. Characteristics included sex and age, genotype $(\Delta \mathrm{F} 508 / \Delta \mathrm{F} 508$ homozygosity), use of pancreatic enzymes and presence of chronic Pseudomonas aeruginosa in lung secretions (defined as 2 or more isolations of Pseudomonas aeruginosa necessitating the use of long term anti-Pseudomonas nebulised antibiotic inhaled therapy).

It was calculated $[14,15]$ that in order to detect a mean difference in FEV1\%pr of $10 \%$ between MI and non-MI groups, a sample size of at least 20 subjects (with each subject having 5 measurements over at least 5 years i.e. 100 observations) is needed for a $5 \%$ significance level at $80 \%$ power.

A random selection of subjects was made from the non-MI group for matched selection purposes by employing the Excel RAND function to provide approximately a 2:1 non-MI:MI ratio.

Primary outcomes were analysed using mixed effects regression models REML (restricted maximum likelihood method). The MI and non-MI groups were compared for each of the main outcomes: FEV1\%pr, FVC\%pr, HtZ and WtZ over time. The models were adjusted for sex, genotype, age of diagnosis, pancreatic status and chronic Pseudomonas aeruginosa pulmonary status. The statistical software package STATA version 10.1 was used. Survival in the two groups was compared using Kaplan-Meier analysis. Graphic representation of the outcomes of lung function and growth was done using MS Excel with the error bars representing the $5^{\text {th }}$ and $95^{\text {th }}$ percentiles around median values for MI and non-MI groups. Subgroup comparison of FEV1\%pr at the age of 10 years was analysed using Mann Whitney (SPSS version 12). 


\section{Results}

From the original 75MI and 590 non-MI subjects registered on the CF database, 38/75 MI subjects were born before $1^{\text {st }}$ January 1993 and had sufficient pulmonary function and anthropometric data (median age of diagnosis 1 day) to meet the above criteria. Of these 38 , thirty underwent a newborn surgical procedure (14 had intestinal resection), 7 subjects were successfully treated with gastrograffin enemas and 1 passed a meconium plug spontaneously at 19 hours of age. 283/ 590 non-MI subjects met the inclusion criteria. From this population of 283, seventy-six subjects were selected at random to act as controls for the MI population (median age of diagnosis 7 months). 30 of these presented with a history of combined chest problems and failure to thrive, 16 with chest problems alone, 15 failure to thrive alone and 15 with other modes.

The Royal Brompton Hospital CF database contained up to date data for all included subjects. The database is complete in that as far as we are aware, if a patient has been seen in the annual clinic, the data is registered on the database. However, not all included patients had data recorded from each annual review. From the 8-year review onwards, on average lung function data for $86 \%$ non-MI and $81 \%$ MI patients was recorded annually. From 2 to 8 years, at each review on average only $60 \%$ nonMI and 56\% MI patients had anthropometric measurements recorded. We believe that missing data is largely due to late referral to the Brompton Hospital and non-attendance at annual appointments.

Since the age of 15 , five subjects from the non-MI group have subsequently died compared to none from the MI group (by $1^{\text {st }}$ January 2009). This difference was not statistically different. (See figure 1).

There was no significant difference between MI and non-MI groups in terms of genotype, sex, median date of birth and median age of first attendance at annual review clinic (table 1). There was also no difference in chronic Pseudomonas colonisation (defined as 2 or more previous sputum isolations of Pseudomonas aeruginosa and on long term nebulised anti-Pseudomonal antibiotics) or in the median age of first sputum isolation of Pseudomonas aeruginosa, There was no significant difference in pancreatic enzyme use. We did not make pancreatic sufficiency an exclusion criteria because, although rare, patients who are pancreatic sufficient can get MI. In order to reflect the CF population, the 6 pancreatic sufficient non-MI patients were therefore included. There was a significant difference, 
however, in the median age of commencing pancreatic enzymes (1 day old in the MI group versus 211 days old in the non-MI group, $\mathrm{p}=0.00$ ).

Figures 2 to 5 display graphically how the median values of all 4 primary outcomes vary over time for both MI and non-MI groups. Figure 2 shows a decline over time for both groups in median FEV1\%pr but the median values are higher in the MI group at all but 1 time point ( 8 years). Figure 3 shows the decline over time of median FVC\%pr in both groups over the study period but with values higher in the MI group at all time points. Figure 4 shows the median HtZ score decreasing over the time period of the study in both groups. Again, the median values are higher at all time points in the MI group apart from 1 (10 years). Figure 5 shows higher median values for $\mathrm{WtZ}$ score at all but 2 time points in the MI group (6 and 12 years), however the overall linear trend is for the median WtZ to decrease in the MI group but increase in the non-MI group over time.

Table 2 shows a statistical comparison of MI and non-MI groups for all 4 outcomes over the time period of the study. The mixed effects REML regression model, adjusting for sex, age of diagnosis, genotype, pancreatic status and presence of chronic Pseudomonas aeruginosa infection and age of diagnosis showed no statistically significant difference between the 2 groups for all 4 parameters over the time period of the study. There was a non-statistically significant trend for the FEV1\%pr to be higher on average by $3.36 \%$ per year in the MI group compared to the non-MI group. Similar trends were seen for the other outcome measures $(2.87 \%$ per year for $\mathrm{FVC} \% \mathrm{pr}, 0.06 \mathrm{HtZ}$ per year and 0.01 $\mathrm{WtZ}$ per year in favour of the MI group).

There is a possibility that over the time course of the study prognosis and survival has improved which could influence the results. We therefore compared the FEV1\%pr at the age of 10 years between MI and non-MI subjects in 2 subgroups, those born < 01/01/1989 and those born between 02/01/1989 and 01/01/1993. In the earlier subgroup, at the age of 10 years, FEV1 was recorded for 27/33 non-MI subjects with a median FEV1\%pr of $83 \%$. This was compared to 13/16 MI subjects with a median FEV1\%pr of $96 \%$. This difference, however, was not statistically significant ( $\mathrm{p}=0.260$, Mann Whitney). In the later subgroup, at the age of 10 years, 43/43 non-MI subjects had a median FEV1\%pr 
of $89 \%$. This compared to $94 \%$ in MI subjects $(n=20 / 22)$. This difference again was not significant ( $\mathrm{p}=0.114$, Mann Whitney). 


\section{Discussion}

Here, we report that during the first 15 years of life there was no statistically significant difference in lung function and growth outcomes between children presenting with meconium ileus and those presenting later with other symptoms during an era of non-NBS. There was a non-significant trend for the prognosis of the MI group to be slightly better than the non-MI group in terms of growth and lung function. This is despite the fact that many of the meconium ileus group had an invasive procedure in the newborn period, which is likely to predispose to acquisition of Psuedomonas aeruginosa. [16], (Our results did not demonstrate this however with MI subjects having the same timing of first Pseudamonas colonisation and rate of chronic infection as non-MI subjects). The significant difference in the median age of starting pancreatic enzymes ( 1 day MI versus 211 days non-MI) is most likely a direct result of earlier diagnosis.

There were more deaths in the non-MI group compared to the MI group though this difference was not statistically significant.

The strengths of this study include the longitudinal design, and the fact that anthropometric, lung function measurements and $\mathrm{CF}$ treatment after the correction of MI was standardised in a single centre. The main weakness is the study size. The inclusion criteria ensured sufficient longitudinal data to power the study but also meant that the final sample excluded patients with fewer data points. Longer follow-up would have been more ideal but this was difficult due to referral of many patients to other adult tertiary centres. However the fact that up to 15 years after surgery (in the MI group) we could not detect a difference between the two groups in the major prognostic factors (spirometry, nutrition) is reassuring,

There is also the possibility, however, that over the 15 -year time course of the study prognosis and survival has improved which could influence the results. However, when 2 subgroups were analysed separately based on year of birth $(<01 / 01 / 89$ or $02 / 01 / 89-01 / 01 / 93)$ there was no significant difference in FEV1\%pr at age 10 years between MI and non-MI subjects. However, drawing any conclusions from this is difficult due to the small numbers compared. 
An alternative study design would be to use a large national database, which would enable larger numbers to be studied. This approach is not without problems; treatment protocols vary from centre to centre, and for most if not all databases, there is no attempt to quality control the anthropometric and of particular importance, the lung function measurements. In a single centre study such as ours there is careful calibration of equipment and standardisation of measurement techniques.

Our findings support our original hypothesis that with current standards of medical care and in an era of non-new born screening, there is no difference in clinical outcome between CF patients presenting with MI and patients without MI presenting with other symptoms.

Our conclusion correlates with that of the study by Munck et al detailed earlier who also compared, on a smaller scale, MI presenting patients to a non-screened population presenting with other symptoms. [8]. It also agrees with the findings of Kappler et al [10], who, over a variable study period, showed no difference in lung function between MI and non-MI non-screened subjects, both on longitudinal and cross-sectional analyses and no difference in nutritional parameters on cross-sectional analysis. Our results also correlate with the $2^{\text {nd }}$ cohort of patients (diagnosed between 1973 and 1982) in the study by Kerem et al in which there was no significant difference between MI and non-MI non-screened groups in terms of lung function and growth.

Our results disagree with those studies comparing MI presenting patients to those without MI diagnosed by new born screening $[4,5,6,7]$. These studies have all shown a worse outcome for MI as compared to non-MI NBS patients. The Wisconsin CF Neonatal Screening Project prospectively compared 32 MI to 50 non-MI patients diagnosed by NBS from diagnosis to twelve years of age [5, 6,7]. They showed that MI patients had significantly worse lung function as well as growth over the study period. Evans et al [4] compared 39 MI with 39 non-MI NBS patients at just one time point. They showed no difference in growth between the two groups but the MI group had significantly worse FEV1\%pr. These studies would suggest that, in these centres at least, either MI per se, or more likely, the effects of interventions in the newborn period, carry a long-term adverse effect. 
It is recognised by some $[17,18,19,20]$ but not by others $[21,22]$ that earlier diagnosis is beneficial to long term clinical outcome in CF. The likely explanation for our findings differing from findings in studies using NBS patients as a control group [4, 5, 6, 7] is that although many of our MI subjects required surgery, the earlier diagnosis of the MI group compared to the non-MI group allowed earlier institution of CF therapy. This may have offset any possible disadvantage to the MI group of invasive interventions in the newborn period. This delay in diagnosis is not as apparent when comparing MIpresenting patients to non-MI patients diagnosed soon after birth by NBS.

In summary, we have shown that within a non-screened population CF patients presenting with MI have the same prognosis (up until the age of 15 years) as those presenting later with other symptoms. Comparing our results to studies involving non-MI NBS subjects as controls, lends support to the benefits of early diagnosis and, indirectly, NBS. Future studies should focus on the outcomes of MI patients compared with non-MI patients diagnosed through NBS, to determine if presenting with MI truly affects long-term outcome in CF in the modern era of surgery and intensive care. If this is the case, there will be a need to focus on improving the peri-operative care of these patients. 


\begin{abstract}
Acknowledgements
The authors would like to thank Jackie Francis, Specialist CF Sister who is responsible for inputting data into the Royal Brompton Hospital CF database and to Winston Banya - Statistician for his statistical expertise.
\end{abstract}




\section{References}

1. Ziegler MM. Meconium ileus. Curr Probl Surg. 1994; 31: 731-777.

2. Mushtaq I, Wright VM, Drake DP, Mearns MB, Wood CB. Meconium ileus secondary to cystic fibrosis. The East London experience. Pediatr Surg Int. 1998; 13 (5-6): 365-369.

3. Fakhoury K, Durie PR, Levison H, Canny GJ. Meconium ileus in the absence of cystic fibrosis. Arch Dis Child 1992; 67: 1204-6

4. Evans AK, Fitzgerald DA, McKay KO. The impact of meconium on the clinical course of children with cystic fibrosis. Pediatr Pulmonol 2004; 38 (4): 277-284.

5. Li Z, Lai HJ, Kosorok MR, Laxova A, Rock MJ, Splaingrd ML, Farrell PM. Longitudinal pulmonary status of cystic fibrosis children with meconium ileus. Pediatr 2005; 147 (3 Suppl): S30-36.

6. Lai H-J, Cheng Y, Cho H, Kosorok MR, Farrell PM. Association between initial disease presentation, lung disease outcomes and survival in patients with cystic fibrosis. Am J Epidemiology 2004; 159: 537-546.

7. Lai H-C, Kosorok MR, Laxova A, Davis LA, FitzSimmon SC, Farrell PM. Nutritional status of patients with cystic fibrosis with meconium ileus: a comparison with patients without meconium ileus diagnosed early through neonatal screening. Pediatrics 2000; 105: 53-61.

8. Munck A, Gerardin M, Alberti C, Ajzenman C, Lebourgeois M, Aigrain Y, Navarro J. Clinical outcome of cystic fibrosis presenting with or without meconium ileus: a matched cohort study. J Pediatr Surg 2006; 41: 1556-1560.

9. Kerem E, Corey M, Kerem B, Durie P, Tsui L, Levison H. Clinical and genetic comparisons of patients with cystic fibrosis, with or without meconium ileus. J Pediatr May 1989; 114(5): 767-73.

10. Kappler M, Feilcke M, Schroter C, Kraxner A, Griese M. Long-term pulmonary outcome after meconium ilues in cystic fibrosis. Pediatr Pulmonol Dec 2009; 44(12): 1201-6.

11. Wald NJ, Morris JK. Neonatal screening for cystic fibrosis. BMJ 1998; 316: 404-5

12. Rosenthal M, Bain SH, Cramer D, Denison D, Bush A, Warner JO. Lung function in white children aged 4 to 19 years: I - spirometry. Thorax 1993 Aug; 48(8):794-802

13. Tanner JM Whitehouse RH, Takaishi M. Standards from maturity for height, weight, height velocity and weight velocity: British children 1965 - part 2. Arch Dis Child 1966; 41:613-635 
14. Gore MS, Altman DG. Statistics in Practice. British Medical Journal Publishing Group 1982. ISBN 0727900854

15. Siegal S, Castellan NJ. Non parametric statistics for the behavioural sciences ( $2^{\text {nd }}$ edition). New York: McGraw-Hill 1988

16. Heinzl B, Eber E, Oberwaldner B, Haas G, Zach MS. Effects of inhaled gentamicin prophylaxis on acquisition of Pseudomonas aeruginosa in children with cystic fibrosis: a pilot study. Pediatr Pulmonology 2002; 33:32-7

17. Farrell PM, Lai HJ, Li Z, Kosorok MR, Laxova A, Green CG, Collins J, Hoffman G, Laessig R, Rock MJ, Splaingard ML. Evidence on improved outcomes with early diagnosis of cystic fibrosis through neonatal screening: enough is enough! 2005 Sep; 147 (3 Suppl): S30-36

18. Accurso FJ, Sontag MK, Wagener JS. Complications associated with symptomatic diagnosis in infants with cystic fibrosis. J Pediatr 2005; 147 (3 Suppl): S37-41.

19. Sims EJ, Clark A, McCormick J, Mehta G, Connett G, Mehta A. Cystic fibrosis diagnosed after 2 months of age leads to worse outcomes and requires more therapy. Pediatrics 2007; 119(1): 156-157.

20. Merrelle ME, Schouten JP, Gerritsen J, Dankert-Roelse JE. Influence of neonatal screening and centralised treatment on long-term clinical outcome and survival of CF patients. Eur Respir J. 2001; 18(2): 306-315.

21. Bowling F, Cleghorn G, Chester A, Curran J, Griffin B, Prado J, Francis P, Shepherd R. Neonatal screening for cystic fibrosis. Arch Dis Child 1988; 63: 196-198.

22. Chatfield S, Owen G, Ryley HC, Williams J, Alfaham M, Goodchild MC, Weller P. Neonatal screening for cystic fibrosis in Wales and the West Midlands: clinical assessment after five years of screening. Arch Dis Child 1991;66: 29-33. 
1

2

3

4

5

6

7

8

9

10

11

12

13

14

15

16

17

18

19

20

21

22

23

24

25

26

27

28

29

30

31

32

33

34

35

36

37

38

39

40

41

42

43

44

45

46

47

48

49

50

51

52

53

54

55

56

57

58

59

60

\section{Title page}

Does presenting with meconium ileus affect the prognosis of children with cystic fibrosis?

${ }^{1}$ Jo-Anne Johnson MBChB (drjojohnson@ doctors.org.uk)

${ }^{2}$ Andrew Bush MD (A.Bush@rbh.nthames.nhs.uk)

${ }^{2}$ Roger Buchdahl MD (rbuc@easynet.co.uk)

${ }^{1}$ Wellcome Trust/Cancer Research UK Gurdon Institute, University of Cambridge, Tennis Court Road, Cambridge, CB2 1QN

${ }^{2}$ Royal Brompton and Harefield NHS Trust, Sydney Street, London, SW6 6NP

Correspondence to:

Professor Andrew Bush

Consultant Respiratory Paediatrician,

Royal Brompton Hospital

Sydney Street

London

SW6 3NP

United Kingdom

Fax 01895279581

E-mail a.bush@rbh.nthames.nhs.uk

Research conducted at Royal Brompton Hospital, Sydney Street, London, SW6 6NP

\section{Short Title}

Meconium ileus and outcome in cystic fibrosis 


\begin{abstract}
Summary
It is a matter of debate as to what extent the long-term outcome of cystic fibrosis (CF) is affected by presenting with meconium ileus (MI). We compared long-term clinical outcomes of CF children who presented with MI, to those presenting with other symptoms (non-MI) in an era of non new-bornscreening (NBS).
\end{abstract}

We collected annual lung function data between the ages of 8 to 15 years in terms of percent predicted first second forced expired volume (FEV1\%pr), percent predicted forced vital capacity (FVC\%pr), and between the ages of 2 to 15 years annual height and weight $\mathrm{z}$-scores ( $\mathrm{HtZ}$ and $\mathrm{WtZ}$ respectively) for children attending the Royal Brompton Hospital CF clinic. To be included in the study, subjects had to have at least 5 pulmonary function tests and 5 anthropometric measurements recorded over this period.

$38 \mathrm{MI}$ and 76 non-MI subjects were compared. There were no significant differences in genotype, sex, chronic Pseudomonas infection, or pancreatic enzyme use between the 2 groups. The median age of diagnosis was 1 day (MI) vs. 7 months (non-MI). There was a decline in spirometry and anthropometric variables over the study period for both MI and non-MI groups apart from WtZ score in the non-MI group. Mixed model analysis adjusting for potential confounders including genotype, pancreatic status, sex, chronic Pseudomonas aeruginosa lung infection, and age of diagnosis revealed no difference between the 2 groups in terms of lung function and growth during the time period of the study, however there was a non-significant trend for subjects presenting with MI to do better in all 4 parameters.

We conclude that babies presenting with MI have no worse long-term outcome than those presenting symptomatically later in infancy, despite having undergone invasive procedures in the newborn period. This underscores the importance of early diagnosis and treatment in CF. 


\author{
Keywords \\ Lung function \\ Growth \\ Presentation \\ New born screening \\ Survival
}




\section{Introduction}

Meconium ileus (MI) is characterised by neonatal intestinal obstruction caused by the accumulation of desiccated and inspissated intraluminal meconium[1]. Around $15 \%$ of patients with pancreatic insufficient cystic fibrosis (CF) present with MI [2] but MI is also recorded rarely in normals and pancreatic sufficient CF [3]. It is a matter of debate as to what extent long-term outcome in CF is affected by MI.

Two groups from areas where new born screening (NBS) is established have suggested that patients presenting with MI have a worse prognosis in terms of lung function than those without MI diagnosed through NBS. [4,5,6,7] One of these groups, the Wisconsin group [5,6,7] also found MI patients had poorer growth.

However, 3 studies have suggested that MI patients have the same prognosis as patients presenting with other symptoms, not diagnosed through new-born-screening. Munck et al [8] in 2006 compared 26 children presenting with MI diagnosed with CF at a median age of 1 month to 26 children without MI presenting with other symptoms (unscreened) diagnosed with CF at a median age of 3.5 months. They compared height and weight $\mathrm{z}$ scores at 1,3,6,10 and 15 years of age. They also compared forced vital capacity (FVC) and first second forced expired volume (FEV1) at 6, 10 and 15 years. There was no difference between the two groups in growth or lung function and they concluded that presenting with MI made no difference to clinical outcome compared to presenting with other symptoms.

The second study [9] compared 158 patients presenting with MI to 1175 CF patients presenting with other symptoms again in a pre-new born screening era. They compared 2 subgroups based on date of birth and showed that MI patients born between 1963 and 1972 had lower weight and height percentiles at age 13 years compared to non-MI patients. However, subjects born between 1973 and 1982 showed no difference in height and weight at 13 years. There were also no differences in FVC, FEV1, or forced expiratory flow at $25 \%$ to $75 \%$ of $\mathrm{FVC}\left(\mathrm{FEF}_{25-75)}\right.$ in this subgroup. There was a significantly better survival in the later compared to the earlier cohort, particularly in the MI group in 
the first year of life. This study suggests that advances in medical care especially in the perinatal period improved the prognosis of patients in the MI group.

The merits of the study by Kerem et al [9] include the large sample size and long time period of the study. However, the problem with such a long time period is the changes in medical practice taking place in this time. Although the authors tried to limit these differences by analysing the results in 2 cohorts, this resulted in a lack of pulmonary function test data to power the study.

The most recent study [10] retrospectively compared $60 \mathrm{CF}$ subjects with a history of MI (mean age of diagnosis 0.4 months) to 310 without MI presenting with other symptoms (mean age of diagnosis 21.8 months). Using a combination of cross-sectional and longitudinal analyses, they showed that there was no difference in lung function between the 2 groups. Although this study is more recent, the large age range of the study subjects ( $0-48$ years) means that it is highly likely that there was wide variation over time in the medical care that the patients received.

The aim of our single centre study is to test the hypothesis that with current standards of medical care and in an era of non-NBS there is no difference in clinical outcome between CF patients presenting with MI and patients without MI presenting with other symptoms.

The question has important practical as well as theoretical implications. If patients presenting with MI have the same or better prognosis than those diagnosed later with other symptoms, despite in many cases having had to undergo a major abdominal operation, this would be a pointer to the benefits of early diagnosis of CF, which has been disputed by some [11]. On the other hand, if they do worse, despite modern treatment, this implies that new treatment strategies are needed in this group and that they would have potentially more to gain from novel, disease-modifying therapies. 


\section{Material and Methods}

Study Subjects:

CF patient data is updated on the Royal Brompton Hospital CF database after the patients' annual review by the CF nurse or secretary. By the $1^{\text {st }}$ January 2008,665 subjects had been registered onto the database since it began in 1986. All had a diagnosis of CF confirmed with a positive sweat test (a chloride value $>60 \mathrm{mmol} / \mathrm{l}$ in two sweat samples) and/or had the presence of two recognised CF disease-causing genetic mutations. Of the 665 subjects 75 of these had presented with meconium ileus and 590 with non meconium ileus (non-MI) symptoms including respiratory symptoms and/or failure to thrive. To be included, subjects had to be at least 15 years old at the time of the study (i.e. born before $1^{\text {st }}$ January 1993). Subjects had to have a minimum of 5 recorded annual pulmonary function tests in terms of percent predicted first second forced expired volume (FEV1\%pr) and percent predicted forced vital capacity (FVC\%pr) from the age of 8 years. They were also required to have at least 5 recordings of annual height and weight $\mathrm{z}$-scores ( $\mathrm{HtZ}$ and $\mathrm{WtZ}$ respectively) from the age of 2 years. Pulmonary and anthropometric data was analysed up to and including the $15^{\text {th }}$ year of life. Ethical approval was obtained from the Royal Brompton Hospital Research Ethics Committee. From 16 years of age many of the subjects were referred to other adult CF tertiary centres so survival data was the only outcome measure recorded thereafter. These were obtained by telephone contact to the adult tertiary centres to which the children had been referred.

Study Design:

The primary objective of our study was to test the hypothesis that, in an era of non-NBS there is no difference in clinical outcome between CF patients presenting with MI (MI group) and those without MI presenting with other symptoms (non-MI group). We used a retrospective longitudinal study to compare growth and pulmonary function data of eligible subjects in MI and non-MI groups on a yearby-year basis. Details of treatment protocols can be found at http://www.rbht.nhs.uk/healthprofessionals/clinical-depts/paediatrics/childrencf/

Methods/Statistical Analysis:

The primary outcomes were annual HtZ /WtZ scores (age 2-15 years) and FVC\%pr/ FEV1\%pr (age 815 years). 8 years was taken as the age at which pulmonary function testing could be accurately 
reproduced. Pulmonary function was measured using a Jaeger CompactLab pneumotachograph in the lung function laboratory by trained technicians. FEV1and FVC were measured and expressed as percentages of predicted values obtained from equations based on gender and height by Rosenthal et al [12]. Weight (minimal underclothing) and height (Harpenden stadiometer) were measured and expressed as standard deviation scores (Z-scores) using published population means and standard deviations [13]. Baseline characteristics of the study groups were compared using Chi Squared analysis with Fisher's exact test for categorical data and Mann-Whitney for continuous data. Characteristics included sex and age, genotype $(\Delta \mathrm{F} 508 / \Delta \mathrm{F} 508$ homozygosity), use of pancreatic enzymes and presence of chronic Pseudomonas aeruginosa in lung secretions (defined as 2 or more isolations of Pseudomonas aeruginosa necessitating the use of long term anti-Pseudomonas nebulised antibiotic inhaled therapy).

It was calculated $[14,15]$ that in order to detect a mean difference in FEV1\%pr of $10 \%$ between MI and non-MI groups, a sample size of at least 20 subjects (with each subject having 5 measurements over at least 5 years i.e. 100 observations) is needed for a $5 \%$ significance level at $80 \%$ power.

A random selection of subjects was made from the non-MI group for matched selection purposes by employing the Excel RAND function to provide approximately a 2:1 non-MI:MI ratio.

Primary outcomes were analysed using mixed effects regression models REML (restricted maximum likelihood method). The MI and non-MI groups were compared for each of the main outcomes: FEV1\%pr, FVC\%pr, HtZ and WtZ over time. The models were adjusted for sex, genotype, age of diagnosis, pancreatic status and chronic Pseudomonas aeruginosa pulmonary status. The statistical software package STATA version 10.1 was used. Survival in the two groups was compared using Kaplan-Meier analysis. Graphic representation of the outcomes of lung function and growth was done using MS Excel with the error bars representing the $5^{\text {th }}$ and $95^{\text {th }}$ percentiles around median values for MI and non-MI groups. Subgroup comparison of FEV1\%pr at the age of 10 years was analysed using Mann Whitney (SPSS version 12). 


\section{Results}

From the original 75MI and 590 non-MI subjects registered on the CF database, 38/75 MI subjects were born before $1^{\text {st }}$ January 1993 and had sufficient pulmonary function and anthropometric data (median age of diagnosis 1 day) to meet the above criteria. Of these 38 , thirty underwent a newborn surgical procedure (14 had intestinal resection), 7 subjects were successfully treated with gastrograffin enemas and 1 passed a meconium plug spontaneously at 19 hours of age. 283/ 590 non-MI subjects met the inclusion criteria. From this population of 283, seventy-six subjects were selected at random to act as controls for the MI population (median age of diagnosis 7 months). 30 of these presented with a history of combined chest problems and failure to thrive, 16 with chest problems alone, 15 failure to thrive alone and 15 with other modes.

The Royal Brompton Hospital CF database contained up to date data for all included subjects. The database is complete in that as far as we are aware, if a patient has been seen in the annual clinic, the data is registered on the database. However, not all included patients had data recorded from each annual review. From the 8-year review onwards, on average lung function data for $86 \%$ non-MI and $81 \%$ MI patients was recorded annually. From 2 to 8 years, at each review on average only $60 \%$ nonMI and 56\% MI patients had anthropometric measurements recorded. We believe that missing data is largely due to late referral to the Brompton Hospital and non-attendance at annual appointments.

Since the age of 15 , five subjects from the non-MI group have subsequently died compared to none from the MI group (by $1^{\text {st }}$ January 2009). This difference was not statistically different. (See figure 1).

There was no significant difference between MI and non-MI groups in terms of genotype, sex, median date of birth and median age of first attendance at annual review clinic (table 1). There was also no difference in chronic Pseudomonas colonisation (defined as 2 or more previous sputum isolations of Pseudomonas aeruginosa and on long term nebulised anti-Pseudomonal antibiotics) or in the median age of first sputum isolation of Pseudomonas aeruginosa, There was no significant difference in pancreatic enzyme use. We did not make pancreatic sufficiency an exclusion criteria because, although rare, patients who are pancreatic sufficient can get MI. In order to reflect the CF population, the 6 pancreatic sufficient non-MI patients were therefore included. There was a significant difference, 
Figures 2 to 5 display graphically how the median values of all 4 primary outcomes vary over time for both MI and non-MI groups. Figure 2 shows a decline over time for both groups in median FEV1\%pr but the median values are higher in the MI group at all but 1 time point ( 8 years). Figure 3 shows the decline over time of median FVC\%pr in both groups over the study period but with values higher in the MI group at all time points. Figure 4 shows the median HtZ score decreasing over the time period of the study in both groups. Again, the median values are higher at all time points in the MI group apart from 1 (10 years). Figure 5 shows higher median values for $\mathrm{WtZ}$ score at all but 2 time points in the MI group ( 6 and 12 years), however the overall linear trend is for the median WtZ to decrease in the MI group but increase in the non-MI group over time.

Table 2 shows a statistical comparison of MI and non-MI groups for all 4 outcomes over the time period of the study. The mixed effects REML regression model, adjusting for sex, age of diagnosis, genotype, pancreatic status and presence of chronic Pseudomonas aeruginosa infection and age of diagnosis showed no statistically significant difference between the 2 groups for all 4 parameters over the time period of the study. There was a non-statistically significant trend for the FEV1\%pr to be higher on average by $3.36 \%$ per year in the MI group compared to the non-MI group. Similar trends were seen for the other outcome measures (2.87\% per year for FVC\%pr, $0.06 \mathrm{HtZ}$ per year and 0.01 WtZ per year in favour of the MI group).

There is a possibility that over the time course of the study prognosis and survival has improved which could influence the results. We therefore compared the FEV1\%pr at the age of 10 years between MI and non-MI subjects in 2 subgroups, those born < 01/01/1989 and those born between 02/01/1989 and 01/01/1993. In the earlier subgroup, at the age of 10 years, FEV1 was recorded for 27/33 non-MI subjects with a median FEV1\%pr of 83\%. This was compared to 13/16 MI subjects with a median FEV1\%pr of $96 \%$. This difference, however, was not statistically significant ( $\mathrm{p}=0.260$, Mann Whitney). In the later subgroup, at the age of 10 years, $43 / 43$ non-MI subjects had a median FEV1\%pr 
of $89 \%$. This compared to $94 \%$ in MI subjects $(n=20 / 22)$. This difference again was not significant ( $\mathrm{p}=0.114$, Mann Whitney). 


\section{Discussion}

Here, we report that during the first 15 years of life there was no statistically significant difference in lung function and growth outcomes between children presenting with meconium ileus and those presenting later with other symptoms during an era of non-NBS. There was a non-significant trend for the prognosis of the MI group to be slightly better than the non-MI group in terms of growth and lung function. This is despite the fact that many of the meconium ileus group had an invasive procedure in the newborn period, which is likely to predispose to acquisition of Psuedomonas aeruginosa. [16], (Our results did not demonstrate this however with MI subjects having the same timing of first Pseudamonas colonisation and rate of chronic infection as non-MI subjects). The significant difference in the median age of starting pancreatic enzymes ( 1 day MI versus 211 days non-MI) is most likely a direct result of earlier diagnosis.

There were more deaths in the non-MI group compared to the MI group though this difference was not statistically significant.

The strengths of this study include the longitudinal design, and the fact that anthropometric, lung function measurements and $\mathrm{CF}$ treatment after the correction of MI was standardised in a single centre. The main weakness is the study size. The inclusion criteria ensured sufficient longitudinal data to power the study but also meant that the final sample excluded patients with fewer data points. Longer follow-up would have been more ideal but this was difficult due to referral of many patients to other adult tertiary centres. However the fact that up to 15 years after surgery (in the MI group) we could not detect a difference between the two groups in the major prognostic factors (spirometry, nutrition) is reassuring,

There is also the possibility, however, that over the 15 -year time course of the study prognosis and survival has improved which could influence the results. However, when 2 subgroups were analysed separately based on year of birth $(<01 / 01 / 89$ or $02 / 01 / 89-01 / 01 / 93)$ there was no significant difference in FEV1\%pr at age 10 years between MI and non-MI subjects. However, drawing any conclusions from this is difficult due to the small numbers compared. 
An alternative study design would be to use a large national database, which would enable larger numbers to be studied. This approach is not without problems; treatment protocols vary from centre to centre, and for most if not all databases, there is no attempt to quality control the anthropometric and of particular importance, the lung function measurements. In a single centre study such as ours there is careful calibration of equipment and standardisation of measurement techniques.

Our findings support our original hypothesis that with current standards of medical care and in an era of non-new born screening, there is no difference in clinical outcome between CF patients presenting with MI and patients without MI presenting with other symptoms.

Our conclusion correlates with that of the study by Munck et al detailed earlier who also compared, on a smaller scale, MI presenting patients to a non-screened population presenting with other symptoms. [8]. It also agrees with the findings of Kappler et al [10], who, over a variable study period, showed no difference in lung function between MI and non-MI non-screened subjects, both on longitudinal and cross-sectional analyses and no difference in nutritional parameters on cross-sectional analysis. Our results also correlate with the $2^{\text {nd }}$ cohort of patients (diagnosed between 1973 and 1982) in the study by Kerem et al in which there was no significant difference between MI and non-MI non-screened groups in terms of lung function and growth.

Our results disagree with those studies comparing MI presenting patients to those without MI diagnosed by new born screening $[4,5,6,7]$. These studies have all shown a worse outcome for MI as compared to non-MI NBS patients. The Wisconsin CF Neonatal Screening Project prospectively compared 32 MI to 50 non-MI patients diagnosed by NBS from diagnosis to twelve years of age [5, 6,7]. They showed that MI patients had significantly worse lung function as well as growth over the study period. Evans et al [4] compared 39 MI with 39 non-MI NBS patients at just one time point. They showed no difference in growth between the two groups but the MI group had significantly worse FEV1\%pr. These studies would suggest that, in these centres at least, either MI per se, or more likely, the effects of interventions in the newborn period, carry a long-term adverse effect. 
It is recognised by some $[17,18,19,20]$ but not by others $[21,22]$ that earlier diagnosis is beneficial to long term clinical outcome in CF. The likely explanation for our findings differing from findings in studies using NBS patients as a control group [4, 5, 6, 7] is that although many of our MI subjects required surgery, the earlier diagnosis of the MI group compared to the non-MI group allowed earlier institution of CF therapy. This may have offset any possible disadvantage to the MI group of invasive interventions in the newborn period. This delay in diagnosis is not as apparent when comparing MIpresenting patients to non-MI patients diagnosed soon after birth by NBS.

In summary, we have shown that within a non-screened population CF patients presenting with MI have the same prognosis (up until the age of 15 years) as those presenting later with other symptoms. Comparing our results to studies involving non-MI NBS subjects as controls, lends support to the benefits of early diagnosis and, indirectly, NBS. Future studies should focus on the outcomes of MI patients compared with non-MI patients diagnosed through NBS, to determine if presenting with MI truly affects long-term outcome in CF in the modern era of surgery and intensive care. If this is the case, there will be a need to focus on improving the peri-operative care of these patients. 


\begin{abstract}
Acknowledgements
The authors would like to thank Jackie Francis, Specialist CF Sister who is responsible for inputting data into the Royal Brompton Hospital CF database and to Winston Banya - Statistician for his statistical expertise.
\end{abstract}




\section{References}

1. Ziegler MM. Meconium ileus. Curr Probl Surg. 1994; 31: 731-777.

2. Mushtaq I, Wright VM, Drake DP, Mearns MB, Wood CB. Meconium ileus secondary to cystic fibrosis. The East London experience. Pediatr Surg Int. 1998; 13 (5-6): 365-369.

3. Fakhoury K, Durie PR, Levison H, Canny GJ. Meconium ileus in the absence of cystic fibrosis. Arch Dis Child 1992; 67: 1204-6

4. Evans AK, Fitzgerald DA, McKay KO. The impact of meconium on the clinical course of children with cystic fibrosis. Pediatr Pulmonol 2004; 38 (4): 277-284.

5. Li Z, Lai HJ, Kosorok MR, Laxova A, Rock MJ, Splaingrd ML, Farrell PM. Longitudinal pulmonary status of cystic fibrosis children with meconium ileus. Pediatr 2005; 147 (3 Suppl): S30-36.

6. Lai H-J, Cheng Y, Cho H, Kosorok MR, Farrell PM. Association between initial disease presentation, lung disease outcomes and survival in patients with cystic fibrosis. Am J Epidemiology 2004; 159: 537-546.

7. Lai H-C, Kosorok MR, Laxova A, Davis LA, FitzSimmon SC, Farrell PM. Nutritional status of patients with cystic fibrosis with meconium ileus: a comparison with patients without meconium ileus diagnosed early through neonatal screening. Pediatrics 2000; 105: 53-61.

8. Munck A, Gerardin M, Alberti C, Ajzenman C, Lebourgeois M, Aigrain Y, Navarro J. Clinical outcome of cystic fibrosis presenting with or without meconium ileus: a matched cohort study. J Pediatr Surg 2006; 41: 1556-1560.

9. Kerem E, Corey M, Kerem B, Durie P, Tsui L, Levison H. Clinical and genetic comparisons of patients with cystic fibrosis, with or without meconium ileus. J Pediatr May 1989; 114(5): 767-73.

10. Kappler M, Feilcke M, Schroter C, Kraxner A, Griese M. Long-term pulmonary outcome after meconium ilues in cystic fibrosis. Pediatr Pulmonol Dec 2009; 44(12): 1201-6.

11. Wald NJ, Morris JK. Neonatal screening for cystic fibrosis. BMJ 1998; 316: 404-5

12. Rosenthal M, Bain SH, Cramer D, Denison D, Bush A, Warner JO. Lung function in white children aged 4 to 19 years: I - spirometry. Thorax 1993 Aug; 48(8):794-802

13. Tanner JM Whitehouse RH, Takaishi M. Standards from maturity for height, weight, height velocity and weight velocity: British children 1965 - part 2. Arch Dis Child 1966; 41:613-635 
14. Gore MS, Altman DG. Statistics in Practice. British Medical Journal Publishing Group 1982. ISBN 0727900854

15. Siegal S, Castellan NJ. Non parametric statistics for the behavioural sciences ( $2^{\text {nd }}$ edition). New York: McGraw-Hill 1988

16. Heinzl B, Eber E, Oberwaldner B, Haas G, Zach MS. Effects of inhaled gentamicin prophylaxis on acquisition of Pseudomonas aeruginosa in children with cystic fibrosis: a pilot study. Pediatr Pulmonology 2002; 33:32-7

17. Farrell PM, Lai HJ, Li Z, Kosorok MR, Laxova A, Green CG, Collins J, Hoffman G, Laessig R, Rock MJ, Splaingard ML. Evidence on improved outcomes with early diagnosis of cystic fibrosis through neonatal screening: enough is enough! 2005 Sep; 147 (3 Suppl): S30-36

18. Accurso FJ, Sontag MK, Wagener JS. Complications associated with symptomatic diagnosis in infants with cystic fibrosis. J Pediatr 2005; 147 (3 Suppl): S37-41.

19. Sims EJ, Clark A, McCormick J, Mehta G, Connett G, Mehta A. Cystic fibrosis diagnosed after 2 months of age leads to worse outcomes and requires more therapy. Pediatrics 2007; 119(1): 156-157.

20. Merrelle ME, Schouten JP, Gerritsen J, Dankert-Roelse JE. Influence of neonatal screening and centralised treatment on long-term clinical outcome and survival of CF patients. Eur Respir J. 2001; 18(2): 306-315.

21. Bowling F, Cleghorn G, Chester A, Curran J, Griffin B, Prado J, Francis P, Shepherd R. Neonatal screening for cystic fibrosis. Arch Dis Child 1988; 63: 196-198.

22. Chatfield S, Owen G, Ryley HC, Williams J, Alfaham M, Goodchild MC, Weller P. Neonatal screening for cystic fibrosis in Wales and the West Midlands: clinical assessment after five years of screening. Arch Dis Child 1991;66: 29-33. 
Kaplan-Meier cumulative survival for MI and Non-MI group. Censored subjects either still alive or died from non CF cause.

$218 \times 134 \mathrm{~mm}(96 \times 96 \mathrm{DPI})$

John Wiley \& Sons, Inc. 
Table 1 Subject Characteristics

\begin{tabular}{|c|c|c|c|c|}
\hline \multicolumn{2}{|l|}{ Characteristic } & \multirow{2}{*}{$\begin{array}{l}\text { MI }(\mathbf{n}=\mathbf{3 8}) \\
25\end{array}$} & \multirow{2}{*}{$\begin{array}{l}\text { Non-MI(n=76) } \\
43\end{array}$} & \multirow{2}{*}{$\begin{array}{l}\text { P value } \\
0.53\end{array}$} \\
\hline Genotype & $\Delta \mathrm{F508} / \Delta \mathrm{F} 508$ & & & \\
\hline & Others & 12 (one missing) & 29 (4 missing) & \\
\hline Chronic & Yes & 18 & 37 & 1.00 \\
\hline $\begin{array}{l}\text { Pseudomonas } \\
\text { infection }^{\mathrm{a}}\end{array}$ & No & 20 & 39 & \\
\hline $\begin{array}{l}\text { Median age of first } \\
\text { Pseudomonas } \\
\text { colonisation }^{\text {b }}\end{array}$ & & 5.1 & 5.0 & 0.265 \\
\hline \multirow{2}{*}{$\begin{array}{l}\text { Pancreatic enzyme } \\
\text { use }\end{array}$} & Yes & 38 & 70 & 0.10 \\
\hline & No & 0 & 6 & \\
\hline $\begin{array}{l}\text { Median age } \\
\text { commenced } \\
\text { pancreatic enzymes } \\
\text { (years) }\end{array}$ & & 0.003 & 0.58 & 0.00 \\
\hline \multirow[t]{2}{*}{ Sex } & Male & 16 & 31 & 1.0 \\
\hline & Female & 22 & 45 & \\
\hline \multicolumn{2}{|l|}{ Median date of birth } & $22 / 09 / 1989$ & 05/06/1989 & 0.93 \\
\hline \multicolumn{2}{|c|}{ Median age at diagnosis (months) } & 0.0329 & 7.44 & 0.00 \\
\hline \multicolumn{2}{|c|}{ Median age of first attendance ${ }^{c}$} & 2.02 & 2.87 & 0.35 \\
\hline
\end{tabular}

${ }^{a}$ Defined as 2 or more previous sputum isolations of Pseudomonas aeruginosa and on long term nebulised anti-Pseudomonal antibiotics; ${ }^{\mathrm{b}}$ Defined as median age of first sputum isolation of Pseudomonas aeruginosa; ${ }^{c}$ Median age of first attendance at annual review clinic; MI: subjects presenting with meconium ileus; non-MI: subjects without meconium ileus presenting with other symptoms; n: number of subjects. 


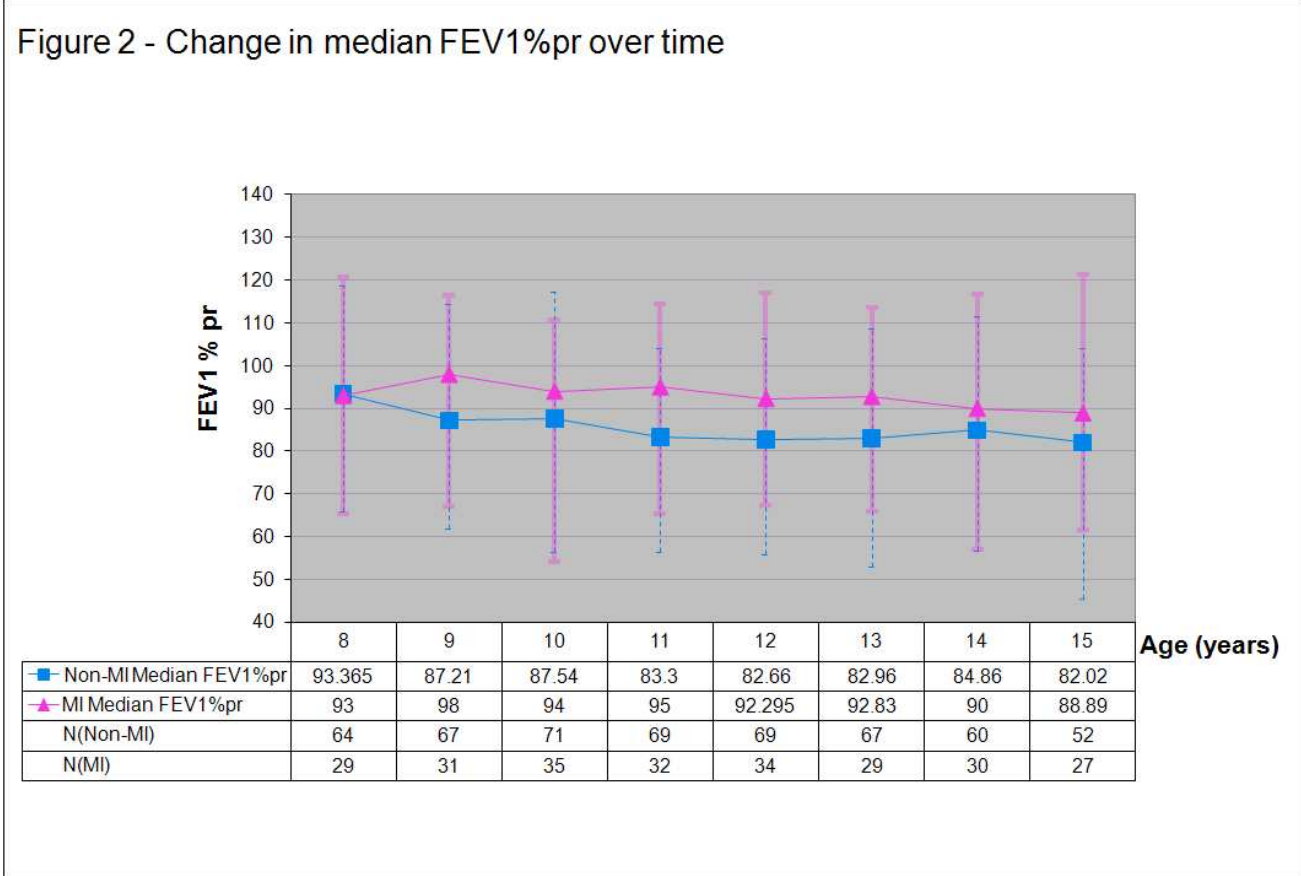

Change in median FEV1\%pr over time in MI versus non-MI groups. Error bars represent 5 th and 95th percentiles for MI and non-MI group median values. FEV1\%pr: percent predicted forced expiratory volume in 1 second; $\mathrm{N}$ : number of subjects; $\mathrm{MI}$ : subjects presenting with meconium ileus; non-MI: subjects without meconium ileus presenting with other symptoms. 


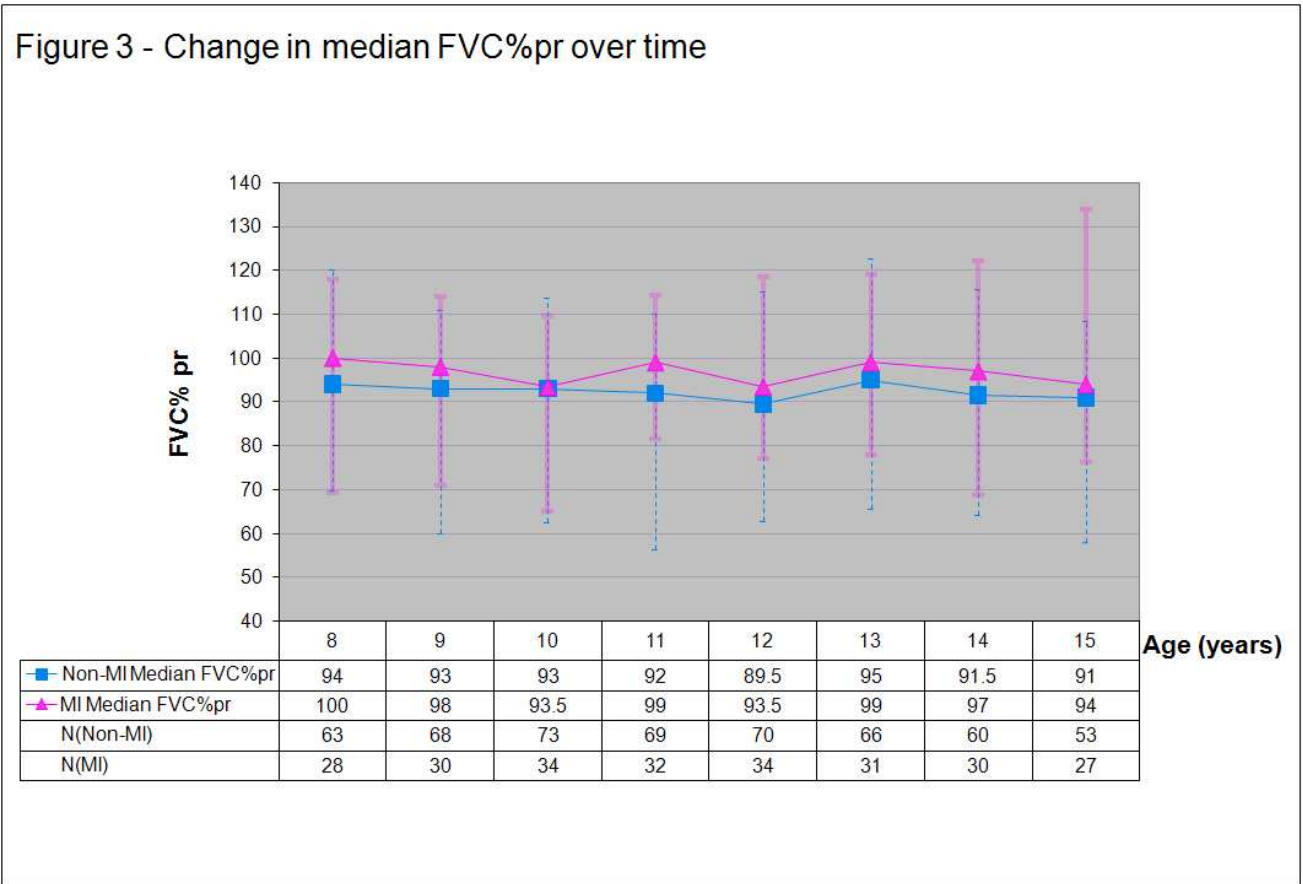

Change in median FVC\%pr over time in MI versus non-MI groups. Error bars represent 5th and 95th percentiles for MI and non-MI group median values. FVC\%pr: percent predicted forced vital capacity; N: number of subjects; MI: subjects presenting with meconium ileus; non-MI: subjects without meconium ileus presenting with other symptoms. 
Change in median $\mathrm{HtZ}$ score over time in MI versus non-MI groups. Error bars represent 5th and 95th percentiles for MI and non-MI group median values. HtZ score: Standard deviation score of height from median; N: number of subjects; MI: subjects presenting with meconium ileus; non-MI: subjects without meconium ileus presenting with other symptoms.

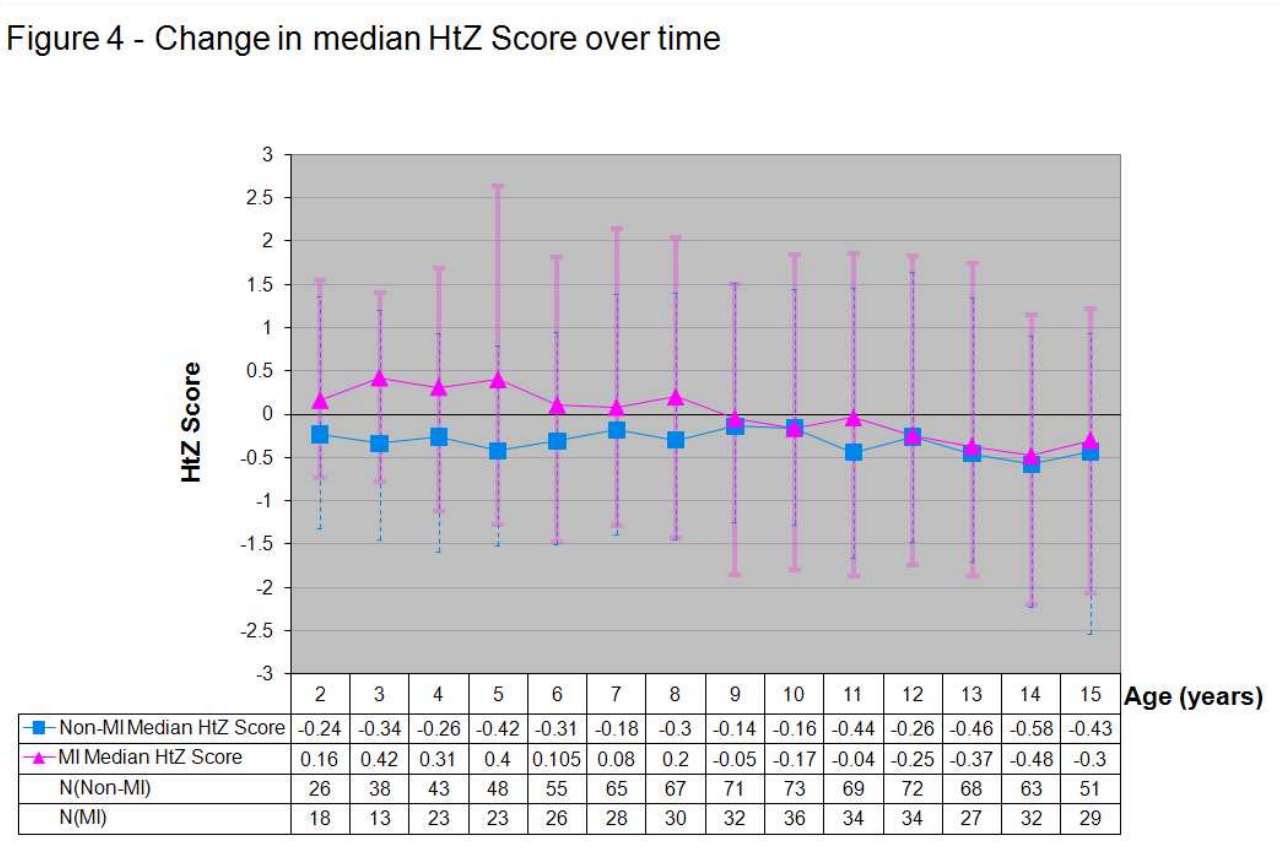




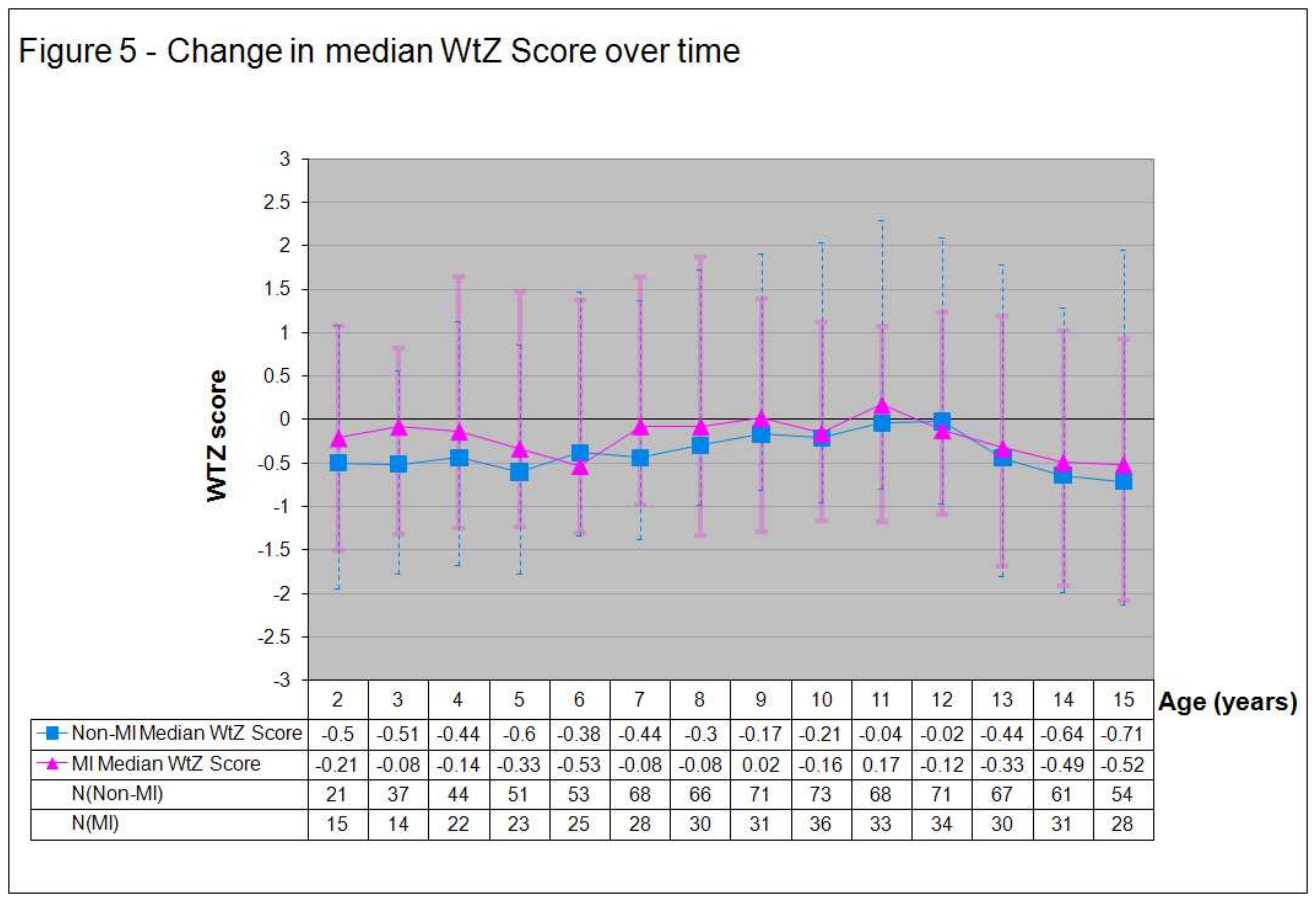

Change in median WtZ score over time in MI versus non-MI groups. Error bars represent 5th and 95th percentiles for MI and non-MI group median values. WtZ score: standard deviation score of weight from median; N: number of subjects; MI: subjects presenting with meconium ileus; non-MI: subjects without meconium ileus presenting with other symptoms. 
2

3

4

5

6

7

8

9

10

11

12

13

14

15

16

17

18

19

20

21

22

23

24

25

26

27

28

29

30

31

32

33

34

35

36

37

38

39

40

41

42

43

44

45

46

47

48

49

50

51

52

53

54

55

56

57

58

59

60

TABLE 2 Mixed effects REML regression model-coefficients for MI versus non-MI subjects

\begin{tabular}{llll}
\hline & Coefficient & P value & 95\% CI \\
\hline FEV1\% pr & 3.36 & 0.11 & -0.71 to 7.42 \\
FVC\% pr & 2.87 & 0.13 & -0.81 to 6.54 \\
HtZ & 0.06 & 0.80 & -0.39 to 1.20 \\
WtZ & 0.01 & 0.96 & -0.35 to 0.38
\end{tabular}

MI: subjects presenting with meconium ileus; non-MI: subjects without meconium ileus presenting with other symptoms; FEV1\%pr: percent predicted first second forced expired volume; FVC\%pr: percent predicted forced vital capacity; HtZ: Height z-score; WtZ: Weight z-score 